\title{
CORRELATED ANISOTROPIES IN THE COSMIC FAR-INFRARED BACKGROUND DETECTED BY THE MULTIBAND IMAGING PHOTOMETER FOR SPITZER: CONSTRAINT ON THE BIAS
}

\author{
G. Lagache, ${ }^{1}$ N. Bavouzet,${ }^{1}$ N. Fernandez-Conde, ${ }^{1}$ N. Ponthieu, ${ }^{1}$ T. Rodet,${ }^{2}$ H. Dole, ${ }^{1}$ \\ M.-A. Miville-Deschênes, ${ }^{1}$ AND J.-L. Puget ${ }^{1}$ \\ Received 2007 May 4; accepted 2007 July 2; published 2007 August 6
}

\begin{abstract}
We report the detection of correlated anisotropies in the cosmic far-infrared background at $160 \mu \mathrm{m}$. We measure the power spectrum in the SWIRE Lockman Hole field. It reveals unambiguously a strong excess above cirrus and Poisson contributions, at spatial scales between $5^{\prime}$ and $30^{\prime}$, interpreted as the signature of infrared galaxy clustering. Using our model of infrared galaxy evolution, we derive a linear bias $b=1.74 \pm 0.16$. It is a factor 2 higher than the bias measured for the local IRAS galaxies. Our model indicates that galaxies dominating the $160 \mu \mathrm{m}$ correlated anisotropies are at $z \sim 1$. This implies that infrared galaxies at high redshifts are biased tracers of mass, unlike in the local universe.
\end{abstract}

Subject headings: galaxies: evolution — infrared: galaxies — large-scale structure of universe

Online material: color figures

\section{INTRODUCTION}

The discovery of the cosmic far-infrared background (CFIB) in 1996, together with recent cosmological surveys from the mid-infrared to the millimeter, has revolutionized our view of star formation at high redshifts. It has become clear, in the last decade, that infrared galaxies contribute to a large part of the whole galaxy buildup in the universe. Since the discovery of the CFIB, new results on the identification of the sources contributing to the CFIB, their redshift distribution, and their nature are coming out at increasing speed, especially through multiwavelength analysis (see for a review Lagache et al. 2005). Stacking analyses are also very promising to probe the CFIB source populations (e.g., Dole et al. 2006; Wang et al. 2006; Dye et al. 2007). However, for now, very little information is available on the clustering of infrared galaxies, although getting information on clustering is essential to our understanding of their formation process and for seeing how they relate to the other galaxy populations.

The first three-dimensional quantitative measurements of the clustering strength of ultra- and hyperluminous infrared galaxies (ULIRGs and HyLIRGs, respectively) at high redshifts $(z>$ 1.5) have been made by Blain et al. (2004), Farrah et al. (2006), and Magliocchetti et al. (2007). These studies show that ULIRGs and HyLIRGs are associated with the most massive dark matter halos at high redshifts, unlike in the local universe where the star formation is quenched in the densest environments. CFIB anisotropy observations provide a powerful complement to direct high angular resolution observations of individual sources. CFIB fluctuations measure the linear clustering bias at large angular scales and the nonlinear clustering within a dark matter halo at small angular scales (Cooray \& Sheth 2002). They thus probe both the dark matter halo mass scale and the physics governing the formation of infrared galaxies within a halo. However, up to now, correlated anisotropies have never been firmly detected. In

\footnotetext{
${ }^{1}$ Institut d'Astrophysique Spatiale (IAS), Bâtiment 121, F-91405 Orsay, France; and Université Paris-Sud 11 and CNRS (UMR 8617), France; guilaine .lagache@ias.u-psud.fr, nicolas.bavouzet@ias.u-psud.fr, nestor.fernandez@ias .u-psud.fr, nicolas.ponthieu@ias.u-psud.fr, herve.dole@ias.u-psud.fr,mamd@ias .u-psud.fr, jean-loup.puget@ias.u-psud.fr.

${ }^{2}$ Laboratoire des signaux et systèmes (L2S), Supélec, 3 rue Joliot-Curie, 91190 Gif-sur-Yvette, France; Thomas.Rodet@1ss.supelec.fr.
}

the far-infrared, detection of anisotropies is limited to the Poisson contribution (Lagache \& Puget 2000; Matsuhara et al. 2000; Miville-Deschênes et al. 2002). ${ }^{3}$

We report the detection of CFIB correlated anisotropies at $160 \mu \mathrm{m}$ in the SWIRE Lockman Hole field and give a first constraint on the linear bias. The Letter is organized as follows: Data are presented in $\S 2$. The power spectrum is analyzed in $\S 3$. Finally, a summary and discussion are given in $\S 4$. Throughout this Letter we use the cosmological parameters $h=0.71, \Omega_{\Lambda}=0.73$, and $\Omega_{m}=0.27$. For the dark matter linear clustering, we set the normalization to $\sigma_{8}=0.8$.

\section{THE LOCKMAN HOLE SWIRE FIELD: MAP AND POWER SPECTRUM}

The Spitzer Wide-Area Infrared Extragalactic survey (SWIRE) has surveyed $49 \mathrm{deg}^{2}$ distributed over six fields in the northern and southern sky (Lonsdale et al. 2004). The Lockman Hole is the largest field with the lowest cirrus emission. It covers about $10 \mathrm{deg}^{2}$ at $160 \mu \mathrm{m}$.

\subsection{Data Reduction and Map}

Raw data were reduced using the Data Analysis Tool (Gordon et al. 2005) version 2.71. We systematically removed each DCE (data collection event) after the stimulator flash to minimize the latency effect. We use the last calibration factor (44.7) to convert MIPS (Multiband Imaging Photometer for Spitzer) units to $\mathrm{MJy} \mathrm{sr}^{-1}$. Data were finally projected on a grid with $15.95^{\prime \prime}$ pixels. The map is shown on Figure 1. Further processings were necessary prior to measuring the power spectrum. We first needed to remove residual stripes. This has been done exactly the same way as in Miville-Deschêne \& Lagache (2005), in which it is shown that our method efficiently removed residual stripes without affecting the astrophysical signal. We then remove all sources with $S_{160}>200 \mathrm{mJy}(200 \mathrm{mJy}$ is the high reliability threshold, as detailed in Surace et al. 2005). For this purpose we use DAOPHOT to detect the sources (the image was wavelet-filtered prior to the detection). We then measure the fluxes using aperture photometry. After a fine

\footnotetext{
${ }^{3}$ Note, however, that Grossan \& Smoot (2006) report the detection of the clustering signature at $160 \mu \mathrm{m}$.
} 

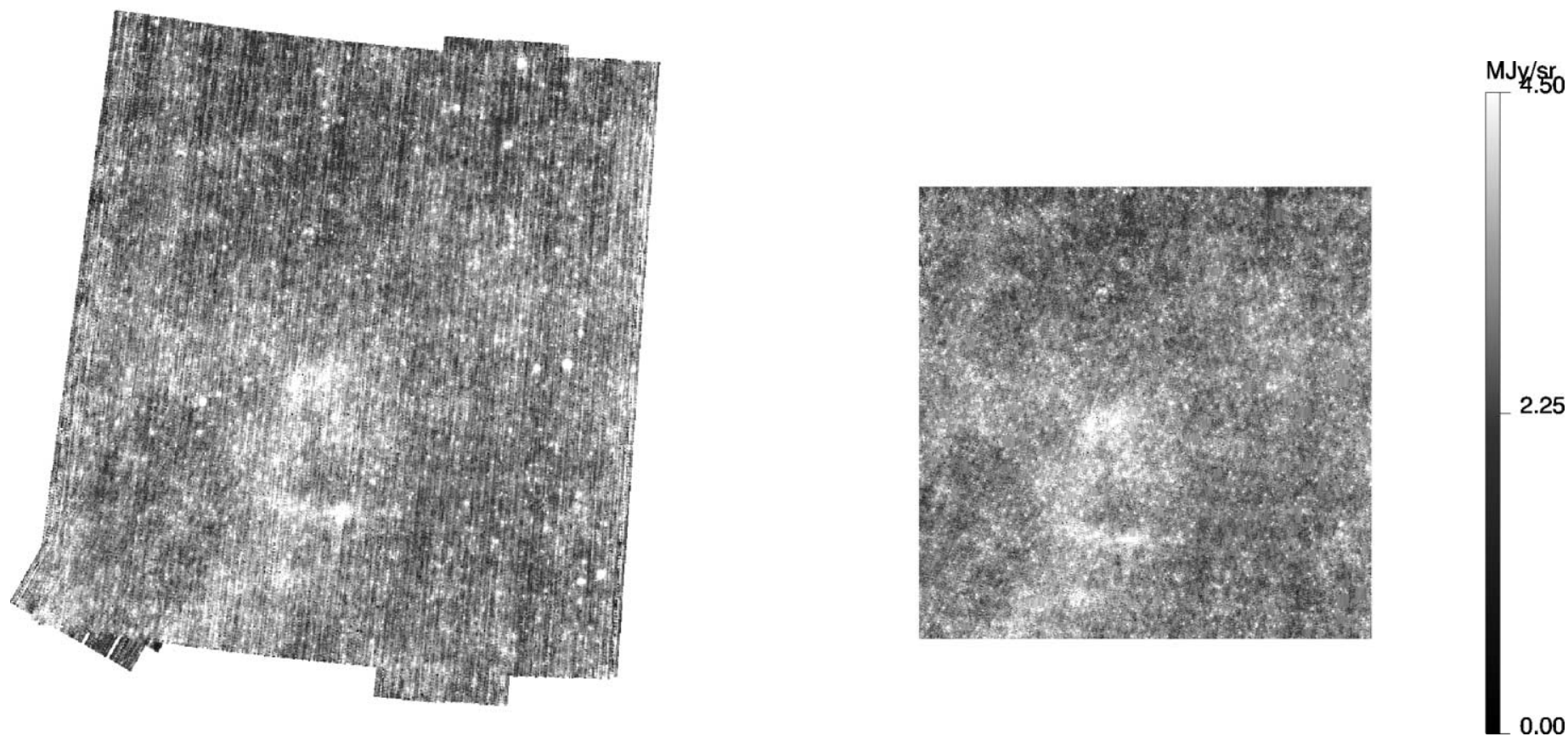

FIG. 1.-Left: $160 \mu \mathrm{m}$ SWIRE map after standard data reduction. Right: Final map used to compute the power spectrum. Residual stripes have been corrected as in Miville-Deschênes \& Lagache (2005). Sources with $S_{160}>200 \mathrm{mJy}$ have been removed. [See the electronic edition of the Journal for a color version of this figure.]

centering on the sources, we integrate within $25^{\prime \prime}$. Sky values are estimated in an $\left[80^{\prime \prime}-110^{\prime \prime}\right]$ annulus. We compute the aperture correction-which is 2.02 - using an effective instrumental function (which we call PSF for point-spread function) measured directly on a MIPS $160 \mu \mathrm{m}$ map. Using an effective PSF rather than the PSF computed using the STinyTim program $^{4}$ is important for taking into account the survey strategy. In the Lockman Hole SWIRE field, the signal-to-noise ratio was not high enough to accurately measure the PSF. We thus use the GTO/CDF-S field in which the integration time is 6

${ }^{4}$ See http://ssc.spitzer.caltech.edu/archanaly/contributed/stinytim/.

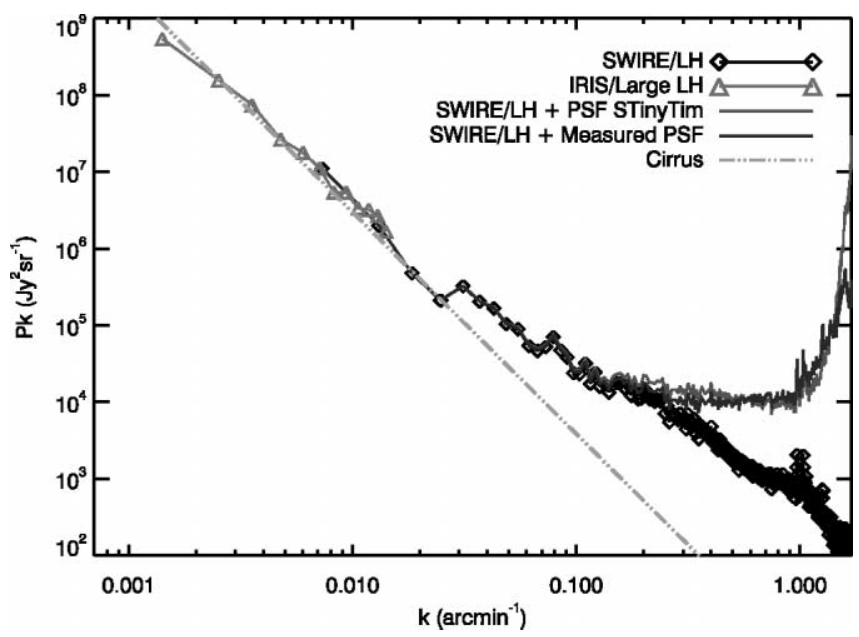

FIG. 2.-Total power spectrum measured in the Lockman Hole (LH) at $160 \mu \mathrm{m}$. The diamonds represent the MIPS/SWIRE $160 \mu \mathrm{m}$ power spectrum $[P(k)-N(k)$; see eq. (1)], and the triangles represent the IRIS/IRAS enlarged Lockman Hole field power spectrum at $100 \mu \mathrm{m}$, scaled to the power spectrum at $160 \mu \mathrm{m}$ using the $160 / 100$ dust color measured at high latitudes $\left(|b|>40^{\circ}\right)$. The gray and black lines represent, respectively, the power spectra corrected from STinyTim and the measured PSF, $[P(k)-N(k)] / \gamma(k)$. The dash-doubledotted line represents the best-fit cirrus power spectrum, as computed in $\S 3.3$. [See the electronic edition of the Journal for a color version of this figure.] times that in the Lockman Hole SWIRE field. We checked that our measured fluxes at $160 \mu \mathrm{m}$ were in very good agreement with the SWIRE DR2 catalog (better than $10 \%$ on average). In Figure 1, we show the final map that will be used to compute the power spectrum.

\subsection{Power Spectra}

There are four contributors to the power spectrum at $160 \mu \mathrm{m}$ : cirrus emission, Poisson (shot) noise from discrete unresolved sources, CFIB clustering (if any), and instrumental noise. If the noise and the signal are not correlated, the measured power spectrum $P(k)$ follows

$$
P(k)=\gamma(k)\left[P_{\text {cirrus }}(k)+P_{\text {source }}+P_{\text {clus }}(k)\right]+N(k),
$$

where $k$ is the two-dimensional wavenumber $\left[k=\left(k_{x}^{2}+k_{y}^{2}\right)^{1 / 2}\right.$, expressed in $\left.\operatorname{arcmin}^{-1}\right], P_{\text {cirrus }}(k), P_{\text {source }}, P_{\text {clus }}(k)$, and $N(k)$ are, respectively, the power spectrum of the dust emission, the shot noise from unresolved sources (constant), the clustering, and the noise. The factor $\gamma(k)$ represents the power spectrum of the PSF. To isolate the astrophysical components, we have to determine $N(k)$ and $\gamma(k)$.

The noise power spectrum $N(k)$ is computed by subtracting two maps of exactly the same region as detailed in MivilleDeschênes et al. (2002). We construct two maps using the even and odd scans. As expected, $N(k)$ and $P(k)$ meet at small scales $\left(k \sim 1 \mathrm{arcmin}^{-1}\right)$ where the signal is noise-dominated. The noise power spectrum $N(k)$ is subtracted from the raw power spectrum $P(k)$.

One of the critical issues is to correct the power spectrum from the PSF $\gamma(k)$. The PSF at $160 \mu \mathrm{m}$ computed using the STinyTim program is very accurate but does not include any effect induced by the observing strategy. We have therefore also extracted the PSF directly from the data (as discussed in $\S 2$ ). A comparison of the corrected power spectrum with these two PSFs is shown in Figure 2. They are in very close agreement, but we notice that the effective PSF gives a better result (i.e., a 
flat power spectrum for $0.25 \operatorname{arcmin}^{-1}<k<0.8 \operatorname{arcmin}^{-1}$, as expected from $\left.P_{\text {source }}\right)$.

The error bars (shown in Fig. 3) are estimated using a frequentist approach. Mock signal-plus-noise maps are generated and analyzed with the same pipeline as for the data. This gives us a set of power spectra from which we compute the covariance matrix. The diagonal elements of the covariance matrix are the errors on the measured power spectrum.

\section{POWER SPECTRUM ANALYSIS}

\subsection{Adding Low Spatial Frequency Data to Constrain the Cirrus Component}

Several studies show that the cirrus component dominates the power spectrum at large scales for $k<0.01 \operatorname{arcmin}^{-1}$ (e.g., Miville-Deschênes et al. 2002, 2007). With the SWIRE data only, in such a low interstellar dust column density field, $P_{\text {cirrus }}$ cannot be constrained. A larger map is needed. We therefore compute the power spectrum of a large $\left(\sim 200 \mathrm{deg}^{2}\right)$ IRIS/IRAS $100 \mu \mathrm{m}$ map (Miville-Deschênes \& Lagache 2005). The SWIRE Lockman Hole field is embedded in this large IRIS map so that the average $100 \mu \mathrm{m}$ dust emission is the same in the SWIRE and in the larger map (4\% difference). Having the same average brightness is important since the normalization of the cirrus power spectrum in the very diffuse region scales as $I_{100}^{2}$ (Miville-Deschênes et al. 2007). We compute the power spectrum of the $100 \mu \mathrm{m}$ map after removing the bright sources, as in Miville-Deschênes et al. (2007). We keep only the largest scales $\left(k<910^{-3} \operatorname{arcmin}^{-1}\right)$, where we have only the contribution from the cirrus component-the CFIB being negligible at these very large scales - and we multiply the power spectrum by the average dust emission color $\left(I_{160} / I_{100}\right)^{2}$. The color has been computed using DIRBE and FIRAS data. We compute the average $|b|>40^{\circ}$ spectrum of the dust emission correlated with the H I gas as in Lagache (2003). We then fit the peak of the dust emission spectrum to get the color. We obtain $I_{160} / I_{100}=2.06$. If we take $|b|>30^{\circ}$, the color varies by $\sim 10 \%$. We show in Figure 2 the $160 \mu \mathrm{m}$ power spectrum derived from IRIS data together with the $160 \mu \mathrm{m}$ MIPS power spectrum. The spectra agree impressively well. We can thus use this extended $P(k)$ to constrain the cirrus contribution. In the following, the two spectra are stitched so that we use one spectrum from $k \sim 0.001$ to $1 \operatorname{arcmin}^{-1}$.

\subsection{Detection of an Excess at Intermediate Scales: Signature of Correlated Anisotropies}

The cirrus component follows

$$
P_{\text {cirrus }}(k)=P_{0}\left(\frac{k}{k_{0}}\right)^{\beta}
$$

where $P_{0}$ is the power spectrum value at $k_{0}=0.01 \operatorname{arcmin}^{-1} . P_{0}$ and $\beta$ are determined by fitting the power spectrum (see $\S 3.3$ ). We obtain $P_{0}=(2.98 \pm 0.66) \times 10^{6} \mathrm{Jy}^{2} \mathrm{sr}^{-1}$ and $\beta=-2.89 \pm$ 0.22 . The normalization can be converted at $100 \mu \mathrm{m}$ using the $160 /$ 100 color given above; we obtain $P_{0}(100 \mu \mathrm{m})=7 \times 10^{5} \mathrm{Jy}^{2} \mathrm{sr}^{-1}$. Considering that the mean cirrus value at $100 \mu \mathrm{m}$ in our field is $I_{100}=0.51 \mathrm{MJy} \mathrm{sr}^{-1}$, our measured $P_{0}(100 \mu \mathrm{m})$ and $\beta$ are in excellent agreement with Miville-Deschênes et al. (2007). The power spectrum of the cirrus component (eq. [2]) is displayed in Figure 2. The measured power spectrum clearly has an excess of power with respect to the cirrus contribution for $k>0.3 \mathrm{arcmin}^{-1}$. We interpret this strong excess as the signature of correlated CFIB anisotropies.

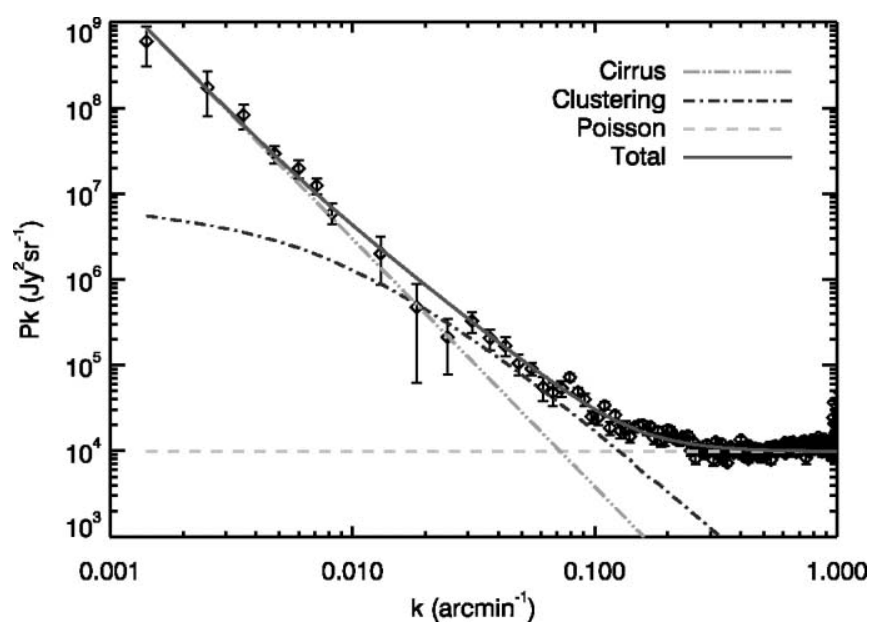

FIG. 3.-Power spectrum in the Lockman Hole at $160 \mu \mathrm{m}$ (diamonds with error bars) with the three components: cirrus (dash-double-dotted line), clustering (dash-dotted line), and Poisson noise (dashed line). The solid line is the sum of the three components. [See the electronic edition of the Journal for a color version of this figure.]

We model the correlated anisotropies following Knox et al. (2001)..$^{5}$ Using the three-dimensional, linear-theory power spectrum of dark matter density fluctuations today, $P_{M}(k)$, the power spectrum of CFIB anisotropies can be written as

$$
C_{l}^{\nu}=\left.\int \frac{d z}{r^{2}} \frac{d r}{d z} a^{2}(z) \overline{j^{2}}(\nu, z) b^{2} P_{M}(k)\right|_{k=l l r} G^{2}(z) \equiv P_{\text {clus }}(k),
$$

where $r$ is the comoving proper-motion distance, $k$ is the threedimensional wavenumber $\left[k=\left(k_{x}^{2}+k_{y}^{2}+k_{z}^{2}\right)^{1 / 2}\right.$, in $\left.\mathrm{Mpc}^{-1}\right]$, $a(z)$ is the scale factor, $\bar{j}(\nu, z)$ is the mean infrared galaxy emissivity per unit of comoving volume, and $G(z)$ is the linear theory growth function; $l$ is the angular multipole sets using the Limber approximation, $k=l / r$. We assume that the fluctuations in emissivity $\delta j / \bar{j}$ are a biased tracer of those in the mass, and we introduce the bias parameter $b$, which we assume to be independent of redshift and scale:

$$
\frac{\delta j(\boldsymbol{k}, \nu, z)}{\bar{j}(\nu, z)}=b \frac{\delta \rho(\boldsymbol{k}, z)}{\bar{\rho}(z)},
$$

where $\rho$ is the dark matter density field. We compute the emissivity using the infrared galaxy evolution model of Lagache et al. (2004). This model, valid in the range 3-1000 $\mu \mathrm{m}$, is in very good agreement with mid-IR to far-IR number counts, CFIB observations, resolved sources' redshift distributions and local luminosity functions, and their evolution up to $z \sim 2$ (e.g., Lagache et al. 2004; Caputi et al. 2006; Dole et al. 2006; Frayer et al. 2006; Caputi et al. 2007). Fixing the cosmology, the only unknown parameter in equation (3) is the bias $b$.

\subsection{Measuring the Bias}

We fit simultaneously $P_{0}, \beta, b$, and $P_{\text {source }}$ using the nonlinear least-squares curve fitting MPFIT program. ${ }^{6}$ We obtain $P_{0}=$ $(2.98 \pm 0.66) \times 10^{6} \quad \mathrm{Jy}^{2} \quad \mathrm{sr}^{-1}, \quad \beta=-2.89 \pm 0.22, \quad b=$ $1.74 \pm 0.16$, and $P_{\text {source }}=9848 \pm 120 \mathrm{Jy}^{2} \mathrm{sr}^{-1} . P_{0}$ and $\beta$ have been discussed in $\S 3.2$. $P_{\text {source }}$ agrees quite well with previous

\footnotetext{
${ }^{5}$ Use of a more complex description of the correlated CFIB anisotropies (by, for example, adding the contribution from the clustering within the same dark matter halo) and the bias is beyond the scope of this Letter.

${ }^{6}$ See http://cow.physics.wisc.edu/ craigm/idl/idl.html.
} 
ISOPHOT determination at roughly the same $S_{160}$ threshold (Matsuhara et al. 2000). The fact that $P_{0}, \beta$, and $P_{\text {source }}$ are in very good agreement with previous measurements gives us confidence in our measurement of the linear bias, $b \sim 1.7$. It is well known that in the local universe, infrared galaxies are not biased tracers of mass. For example, Saunders et al. (1992) found $b \sigma_{8}=0.69 \pm 0.09$ for IRAS galaxies. Assuming $\sigma_{8}=0.8$ gives $b=0.86$. This bias is roughly comparable to the bias of the SDSS galaxies at $z \sim 0.1(b \sim 1.1$; Tegmark et al. 2004). We measure an average bias about 2 times higher in the CFIB anisotropies. Figure 4 shows the predicted redshift contribution to the correlated anisotropies. At $k=0.05 \operatorname{arcmin}^{-1}$, anisotropies from $0.7<z<1.5$ infrared galaxies contribute more than $\sim 65 \%$. Lower redshift galaxies contribute less than $5 \%$. This shows that infrared galaxies at $z \sim 1$ are much more biased ( $\sim 2$ times) than local IRAS galaxies.

\section{SUMMARY AND DISCUSSION}

We presented the power spectrum measured in the SWIRE Lockman Hole field at $160 \mu \mathrm{m}$. It is very well reproduced by the contribution from three components: cirrus, correlated CFIB, and Poisson noise. The cirrus and Poisson contributions are very close to previous measurements. We measure the linear bias, $b=1.74 \pm 0.16$. This bias is likely to be that of infrared galaxies at $z \sim 1$ since $z \sim 1$ galaxies dominate the contribution to the correlated CFIB anisotropies (Fig. 4) and since local galaxies are "antibiased." Such a bias is analogous (but somewhat higher) to the bias of the red optical galaxy population at $z \sim 1$. For example, Marinoni et al. (2005) measured a bias of 1.6 at $z \sim$ 1.2 for the red $(B-I)>1.5$ galaxies in the VIMOS VLT Deep Survey. Blue galaxies at those redshifts are less biased, with a relative bias between red and blue populations of 1.4.

The very strong evolution of the bias (from $\sim 1.7$ at $z \sim 1$ to 0.86 at $z=0$ ) shows that as time progresses and the density field evolves, nonlinear peaks become less rare events, and galaxy formation moves to lower $\sigma$ peaks. Thus, galaxies become less biased tracers of the mass density field. Moreover, for the infrared galaxy population, it is likely that an additional mechanism contributes significantly to the "debiasing" at low redshift. Galaxies in dense environments are found to have suppressed star formation rates (thus no or low infrared emission) and early morphological types compared with those in the field. Environmental effects, in particular, are important in

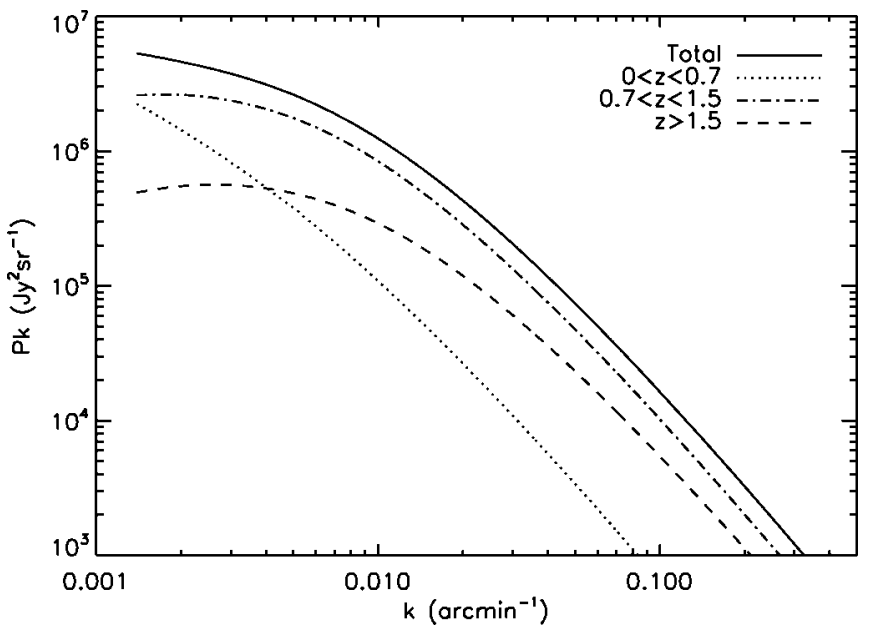

FIG. 4.-Redshift contribution to the correlated anisotropies at $160 \mu \mathrm{m}$ for $b=1.7$.

quenching the star formation through gas stripping (e.g., Postman et al. 2005), although, in some cluster outskirts, some star formation continues (Duc et al. 2002; Coia et al. 2005). The high bias found for infrared galaxies at $z \sim 1$ shows that star formation rates of galaxies are increasing with the environment (as also shown by, e.g., Elbaz et al. 2007). The "merger bias" is an alternative but somehow physically linked way of boosting the bias at high redshift. Clustering of objects that have undergone recent mergers can be enhanced relative to the clustering of individual halos of comparable mass (e.g., Furlanetto \& Kamionkowski 2006; Wetzel et al. 2007). It is known that the star formation in infrared galaxies is triggered to some extent by mergers in dense environments (at $z \sim 1,30 \%-50 \%$ of luminous infrared galaxies are major mergers). Thus, the high measured bias may also point to "merger bias."

This work is based on observations made with the Spitzer Space Telescope, which is operated by the Jet Propulsion Laboratory, California Institute of Technology, under NASA contract 1407. This work benefited from funding from the CNES (Centre National d'Etudes Spatiales) and the PNC (Programme National de Cosmologie). We warmly thank Asantha Cooray for helpful comments and suggestions.

\section{REFERENCES}

Blain, A. W., Chapman, S. C., Smail, I., \& Ivison, R. 2004, ApJ, 611, 725

Caputi, K. I., et al. 2006, ApJ, 637, 727 . 2007, ApJ, 660, 97

Coia, D., et al. 2005, A\&A, 430, 59

Cooray, C. J., \& Sheth, R. 2002, Phys. Rep., 372, 1

Dole, H., et al. 2006, A\&A, 451, 417

Duc, P.-A., et al. 2002, A\&A, 382, 60

Dye, S., Eales, S. A., Ashby, M. L. N., Huang, J.-S., Egami, E., Brodwin, M., Lilly, S., \& Webb, T. 2007, MNRAS, 375, 725

Elbaz, D., et al. 2007, A\&A, 468, 33

Farrah, D., et al. 2006, ApJ, 641, L17 (erratum 643, L139)

Frayer, D. T., et al. 2006, AJ, 131, 250

Furlanetto, S. R., \& Kamionkowski, M. 2006, MNRAS, 366, 529

Gordon, K. D., et al. 2005, PASP, 117, 503

Grossan, B., \& Smoot, G. F. 2006, A\&A, submitted (astro-ph/0604512)

Knox, L., Cooray, A., Eisenstein, D., \& Haiman, Z. 2001, ApJ, 550, 7

Lagache, G. 2003, A\&A, 405, 813

Lagache, G., \& Puget, J.-L. 2000, A\&A, 355, 17

Lagache, G., Puget, J.-L., \& Dole, H. 2005, ARA\&A, 43, 727
Lagache, G., et al. 2004, ApJS, 154, 112

Lonsdale, C., et al. 2004, ApJS, 154, 54

Magliocchetti, M., Silva, L., Lapi, A., de Zotti, G., Granato, G. L., Fadda, D., \& Danese, L. 2007, MNRAS, 375, 1121

Marinoni, C., et al. 2005, A\&A, 442, 801

Matsuhara, H., et al. 2000, A\&A, 361, 407

Miville-Deschênes, M.-A., \& Lagache, G. 2005, ApJS, 157, 302

Miville-Deschênes, M.-A., Lagache, G., Boulanger, F., \& Puget, J.-L. 2007, A\&A, 469, 595

Miville-Deschênes, M.-A., Lagache, G., \& Puget, J.-L. 2002, A\&A, 393, 749

Postman, M., et al. 2005, ApJ, 623, 721

Saunders, W., Rowan-Robinson, M., \& Lawrence, A. 1992, MNRAS, 258, 134

Surace, J. A., et al. 2005, Data Release Paper for DR2 (Pasadena: JPL/NASA), http://swire.ipac.caltech.edu/swire/astronomers/data_access.html

Tegmark, M., et al. 2004, ApJ, 606, 702

Wang, W.-H., Cowie, L. L., \& Barger, A. J. 2006, ApJ, 647, 74

Wetzel, A. R., Cohn, J. D., White, M., Holz, D., \& Warren, M. S. 2007, ApJ, 656,139 